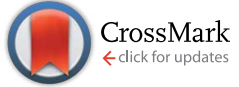

Cite this: RSC Adv., 2016, 6, 38454
Received 6th February 2016

Accepted 8th April 2016

DOI: 10.1039/c6ra03490j

www.rsc.org/advances

\section{Growth kinetics of colloidal Ge nanocrystals for light harvesters $\uparrow$}

\author{
Salvatore Cosentino, ${ }^{* a b}$ Giacomo Torrisi, ${ }^{a}$ Rosario Raciti, ${ }^{a}$ Massimo Zimbone, ${ }^{a}$ \\ Isodiana Crupi, ${ }^{\text {ac }}$ Salvo Mirabella ${ }^{a}$ and Antonio Terrasi ${ }^{a}$
}

Colloidal Ge nanocrystals (NCs) are gaining increased interest because of their potential application in lowcost optoelectronic and light harvesting devices. However, reliable control of colloidal NC synthesis is often an issue and a deeper understanding of the key-role parameters governing NC growth is highly required. Here we report an extended investigation on the growth of colloidal Ge NCs synthesized from a one-pot solution based approach. A systematic study of the effects of synthesis time, temperature and precursor concentration is elucidated in detail. X-ray diffraction (XRD) analysis reveals the presence of crystalline Ge NCs with a mean size (from 5 to $35 \mathrm{~nm}$ ) decreasing with the increase of precursor concentration. Such a trend was further confirmed by scanning electron microscopy (SEM) and dynamic light scattering (DLS) analysis. Moreover, the temporal NC size evolution shows a typical saturating behaviour, where characteristic time shortens at higher precursor concentration. All these growth features were satisfactorily simulated by a numerical NC growth model, evidencing that the kinetics of NC growth is controlled by a reaction-limited regime with typical activation energy of $0.7 \mathrm{eV}$. Finally, light absorption in the visible region and the successful realization of a hybrid photodetector, employing colloidal Ge NCs embedded in PEDOT:PSS polymer, showed the capability of low-cost colloidal Ge to act as light harvester. These results put new understanding for a reliable control of colloidal NC growth and the development of low-cost devices.

\section{Introduction}

Group IV semiconductor nanostructures gained large interest in the last two decades because of their fascinating optical properties and efficient light absorption. ${ }^{\mathbf{1 , 2}}$ In particular, Ge nanostructures (NS) demonstrate a large tuning of optical properties through an optimized exploitation of quantum confinement effects (QCE) and interface/matrix engineering. ${ }^{3-5}$ Such evidences make them very promising for a new class of efficient and tunable optoelectronic devices and light harvesters.

As far as the Ge NS synthesis is concerned, many established methods are commonly used, such as sputtering, chemical vapour deposition, molecular beam epitaxy, ion implantation. ${ }^{6-11}$ Although a solid-phase and high-vacuum based approach is compatible with standard device fabrication route, a low-cost and controlled method would be highly desired for real exploitation of Ge NSs. Indeed, some recent reports have demonstrated successful attempts in the development of

${ }^{a}$ CNR-IMM and Dipartimento di Fisica e Astronomia, Università di Catania, via S. Sofia 64, Catania, Italy.E-mail: salvatore.cosentino@ct.infn.it

${ }^{b}$ Laboratory of Solid State Physics and Magnetism, KU Leuven, B-3001 Leuven, Belgium ${ }^{c}$ Department of Energy, Information engineering and Mathematical models (DEIM), University of Palermo, Viale delle Scienze, Building 9, 90128 Palermo, Italy

$\dagger$ Electronic supplementary information (ESI) available. See DOI: 10.1039/c6ra03490j photodetectors and solar cells using low-cost colloidal nanocrystals (NCs) as active media. ${ }^{\mathbf{1 2 - 1 5}}$ Among low-cost methods, solution based syntheses, represent a powerful method toward the development of cost-effective devices based on Ge NCs. Recently, Vaughn et al. published a comprehensive review outlining known methods for the synthesis of colloidal Ge NCs. ${ }^{\mathbf{1 6}}$ The most common routes include metathesis reactions (double decomposition) involving Zintl salts, ${ }^{17-19}$ hydride reduction of Ge halides, ${ }^{\text {20-24 }}$ thermal decomposition of organo-germane precursors, ${ }^{25,26}$ and one pot heat-up method. In the latter, a Ge salt ( $\mathrm{GeX}, \mathrm{X}=\mathrm{Cl}, \mathrm{Br}, \mathrm{I})$ is typically dissolved and reduced by oleylamine, oleic acid, and hexamethyldisilazane based solutions. ${ }^{27,28}$

These methods are usually able to generate crystalline Ge nanoparticles with some control of the size and/or shape. Despite these successful reports on Ge NC synthesis, a complete understanding and fully reliable control of Ge $\mathrm{NC}$ growth remain still a challenge. In particular, experimental studies showed some controversies in the kinetics of Ge NC growth and their optical properties. For example, Vaughn et al. generated multi-faceted Ge NCs dissolving $\mathrm{GeI}_{4}$ precursor in a mixture solution of oleylamine, oleic acid and hexamethylsilazane at $260{ }^{\circ} \mathrm{C}$ for $30 \mathrm{~min} .{ }^{27}$ By varying the precursor concentration, the NC size was effectively tuned in the 6-22 nm range. However, changing the solvent and varying the surfactant concentration 
leads to completely different morphologies of Ge nanostructures, including cubic-shaped and one dimensional structures. ${ }^{27}$ In a similar synthesis process, Muthuswamy et al. found only slight variations of Ge NC size when the more reactive $\mathrm{GeI}_{2}$ precursor is dissolved in oleylamine, while a better control with the precursor content occurred when a mixture of $\mathrm{GeI}_{2} / \mathrm{GeI}_{4}$ was used. ${ }^{28}$ Indeed, the Ge NC nucleation and growth process does not depend on the precursor concentration only. It was experimentally observed by Codoluto and co-workers that the amount and type of the reducing agent largely influence these processes. ${ }^{29}$ In particular, the chemical environment surrounding Ge NCs plays a key-role also for what concerns the optical properties, being able to influence QCE in both absorption and emission processes..$^{30,31}$

Besides several reports on successful synthesis of Ge nanoparticles from Ge halides, only fewer systematic investigations exist on the effects of the main synthesis parameters on the NC growth. More importantly, a satisfactory interpretation of experimental results based on a proper modeling is still lacking. Up to date, only few reports model exhaustively the growth evolution of colloidal NCs ensembles, ${ }^{32,33}$ while a systematic comparison between experimental results and theoretical calculation was never strengthen. Thus, major gaps between experimental results and theoretical modeling still need to be overcome in order to provide a reliable control of the growth process of colloidal NCs.

For these reasons, here we report an extended investigation on the growth kinetics of colloidal Ge NCs. The experimental results of the effects of synthesis time, temperature and precursor concentration on the growth of Ge NCs are systematically simulated through analytical modelling. These results give evidences that the kinetic of growth is controlled by a reaction-limited regime. Finally, a prototype device for light detection was successfully realized, demonstrating the potential usage of colloidal Ge NC for low-cost optoelectronic devices.

\section{Experimental}

Germanium(II) bromide ( $\mathrm{GeBr}_{2} 97 \%$ pure), oleylamine (70\% technical grade), toluene $(99.8 \%)$, and poly(3,4-ethylenedioxythiophene)-poly(styrenesulfonate) (PEDOT:PSS, 1.3 wt $\%$ dispersion in $\mathrm{H}_{2} \mathrm{O}$, conductive grade) were purchased from Sigma-Aldrich Inc. In our synthesis, $10 \mathrm{ml}$ of oleylamine were purified in a three neck flask by Ar bubbling and stirring for 30 min at $90{ }^{\circ} \mathrm{C}$. Then, $\mathrm{GeBr}_{2}$ salt [from $0.055 \mathrm{~g}(\sim 24 \mathrm{mM})$ to $0.22 \mathrm{~g}$ $(\sim 95 \mathrm{mM})$ ] was added in the solution as a precursor. $\mathrm{GeBr}_{2}$ was dissolved in oleylamine through stirring and heating at $90{ }^{\circ} \mathrm{C}$ for 30 minute under oxygen-free conditions. After complete dissolution of $\mathrm{GeBr}_{2}$, the mixture solution was heated up to 230-260 ${ }^{\circ} \mathrm{C}$ in $\mathrm{Ar}$ atmosphere. The ramp of the heating temperature was controlled at $5{ }^{\circ} \mathrm{C} \mathrm{min}^{-1}$. The dwell time at the final temperature was varied between 1 and $30 \mathrm{~min}$. After this step, the colloidal solution was slowly cooled down to room temperature (ramp at around $10{ }^{\circ} \mathrm{C} \mathrm{min}^{-1}$ ) and $20 \mathrm{ml}$ toluene was added to the mixture in order to disperse Ge NCs better. The presence and size distribution of Ge NCs were investigated through Scanning Electron Microscopy (SEM) and X-Ray Diffraction (XRD) analyses on powders obtained from concentrated Ge NCs solution dried on glass substrates. High resolution SEM analysis was performed with a FEG-SEM Supra 25 microscope, operating at $4 \mathrm{kV}$ on concentrated Ge nanoparticle solution deposited on carbon-coated $\mathrm{Cu}$ grids. Nanoparticles were recognized manually, by locating their boundaries, through GATAN software. For each sample about one hundred particles were analysed and the average size and standard deviation were calculated. XRD analysis was performed using a Bruker D-500 diffractometer on thin films powders deposited by dropping small quantities of colloidal solution on glass substrates. Spectra were acquired in $2 \theta$ glancing incidence mode in the $20-60^{\circ}$ range and with a step resolution of $0.05^{\circ}$. The mean grain size, $S$, of Ge NCs was estimated using the Scherrer equation, ${ }^{34}$ considering the averaged value extracted from the FWHM of Ge crystalline XRD peaks. Vis/NIR transmission spectroscopy was performed in the 400-1100 $\mathrm{nm}$ range on colloidal Ge solutions diluted in toluene (1:3 ratio) by using a Varian Cary 500 spectrophotometer and optically transparent quartz cuvette of $1 \mathrm{~cm}$ optical path length.

The light harvesting performances of Ge NCs were tested in hybrid organic-inorganic devices. Firstly, PEDOT:PSS solution was treated with isopropanol solvation (80\% IPA : $20 \%$ DI water). Such treatment was demonstrated to enhance PEDOT:PSS conductivity, as reported in ref. 35 . As a result of the solvation process PEDOT:PSS film resistance decreased of two order of magnitude, going from $1.6 \times 10^{4} \Omega \mathrm{sq}^{-1}$ to $1.1 \times 10^{2} \Omega$ sq. ${ }^{-1}$. Then, concentrated solution of Ge $\mathrm{NC}$ was added to PEDOT:PSS films and spin (1000 rpm, $30 \mathrm{~s})$ on interdigitated gold electrodes, which were previously fabricated by Au etching photolithography on glass substrates. The imprinted area of the mask on our samples is about $1 \mathrm{~cm}^{2}$ while the interdigitated pattern consists of 9 fingers, separated from each other by a distance of $1 \mathrm{~mm}$. Current vs. time measurements were performed in dark and under illumination with a Keithley 4200 semiconductor characterization system. As light source, we used a fiber optic ring illuminator connected to a $150 \mathrm{~W}$ cold lamp (Highlight 3001, Olympus).

The evolution of the NC growth was studied with a finiteelement-analysis simulation model through COMSOL Multiphysics4.3a. Simulations were performed using an extra-fine complete spatial mesh, considering a single NP with radius $r$ dispersed in a solution having a monomer concentration $C_{\mathrm{Ge}}$ and a unit volume $V$ (defined by the mean distance between nanoparticles).

\section{Results and discussion}

Fig. 1 describes the synthesis process of Ge nanoparticles formed through the dissolution of $0.11 \mathrm{~g} \mathrm{GeBr}_{2}(\sim 47 \mathrm{mM})$ in 10 $\mathrm{ml}$ of oleylamine at $260{ }^{\circ} \mathrm{C}$ for $10 \mathrm{~min}$. Oleylamine is a solvent which plays a double role in the reduction process of $\mathrm{GeBr}_{2}$ and in the stabilization of the Ge NCs. ${ }^{16,36}$ During the heating process, the solution rapidly changes color at about $260{ }^{\circ} \mathrm{C}$, going from a pale yellow (initial stage) to a dark brown color (final stage), as shown in Fig. 1(a) and (b) respectively. The 


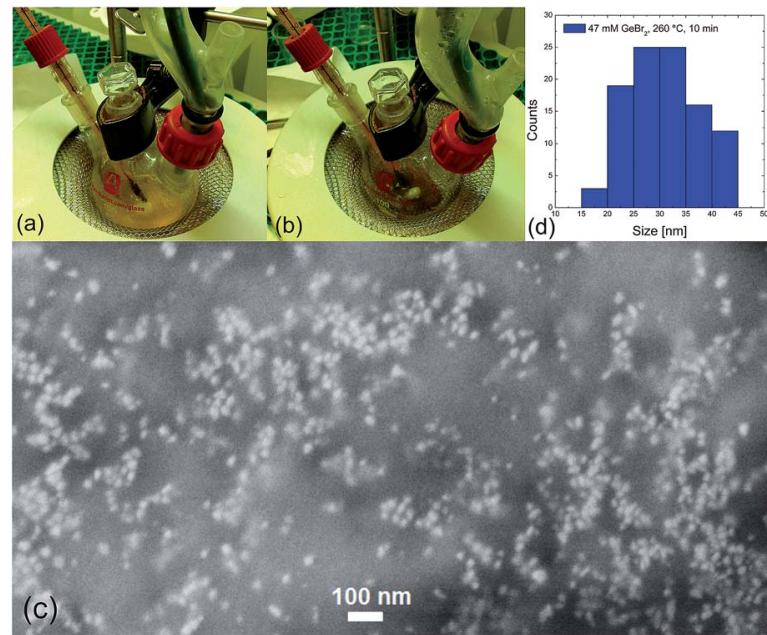

Fig. 1 Color change of colloidal Ge NCs in oleylamine at initial (a) and final (b) stage of synthesis process. Typical SEM micrograph (c), showing Ge nanoparticles with mean size of $30 \pm 5 \mathrm{~nm}$ (d).

color of the system is qualitatively related to the amount of nucleated nanoparticles and to their size. A light-color solution could be either related to a small amount of absorbing centers and/or to the presence of very small nanoparticles having a higher energy absorption onset due to QCE. As soon as the thermal budget is increased, the color of the solution gets darker because of the nucleation of a larger amount of nanoparticles and their simultaneous growth, which turns out in a stronger light absorption capability. A similar behavior was observed also by Xue et al. ${ }^{13}$ and Muthuswamy et al. ${ }^{28,29}$ They interpreted this behavior as a result of the key-role of oleylamine in the reduction process of $\mathrm{GeBr}_{2}$. The authors proposed that the synthesis proceeds firstly with the nucleation of $\mathrm{Ge}(0)$ seeds from the low-temperature reduction of $\mathrm{GeBr}_{2}$, followed by subsequent $\mathrm{Ge}$ nanoparticle growth at higher $T .^{13,37}$ The presence of nanoparticles is verified by the SEM micrograph in Fig. 1(c) and (d), showing Ge nanoparticles having a mean size of $30 \pm 5 \mathrm{~nm}$. Similar values of NC size and dispersion were further checked on an additional sample grown under similar experimental conditions, as reported in Fig. 1S of the ESI. $\dagger$

Indeed, the presence of Ge NCs is also confirmed by XRD analysis performed on powder films obtained from concentrated colloidal solution dried on glass substrates, as shown in Fig. 2. The diffraction peaks at $27.3^{\circ}, 45.4^{\circ}$ and $54.5^{\circ}$ correspond to the $\{111\},\{220\}$ and $\{311\}$ lattice planes of diamond-phase crystalline Ge, respectively. Fig. 2(a) shows the influence of Ge precursor content, $C_{\mathrm{Ge}}$, on the growth process of NCs. The FWHM of Ge crystalline peaks increases with the increasing of Ge content, indicating the presence of smaller NCs. The synthesis time also deeply affects NC growth, as shown in Fig. 2(b). For a synthesis time longer than 1-2 min, clear diffraction peaks from crystalline Ge are present. Moreover, the longer the synthesis time, the sharper FWHM of the peaks is. This behavior evidences a fast reaction process in the NC size growth. On the other hand, no Ge-related crystalline peaks can

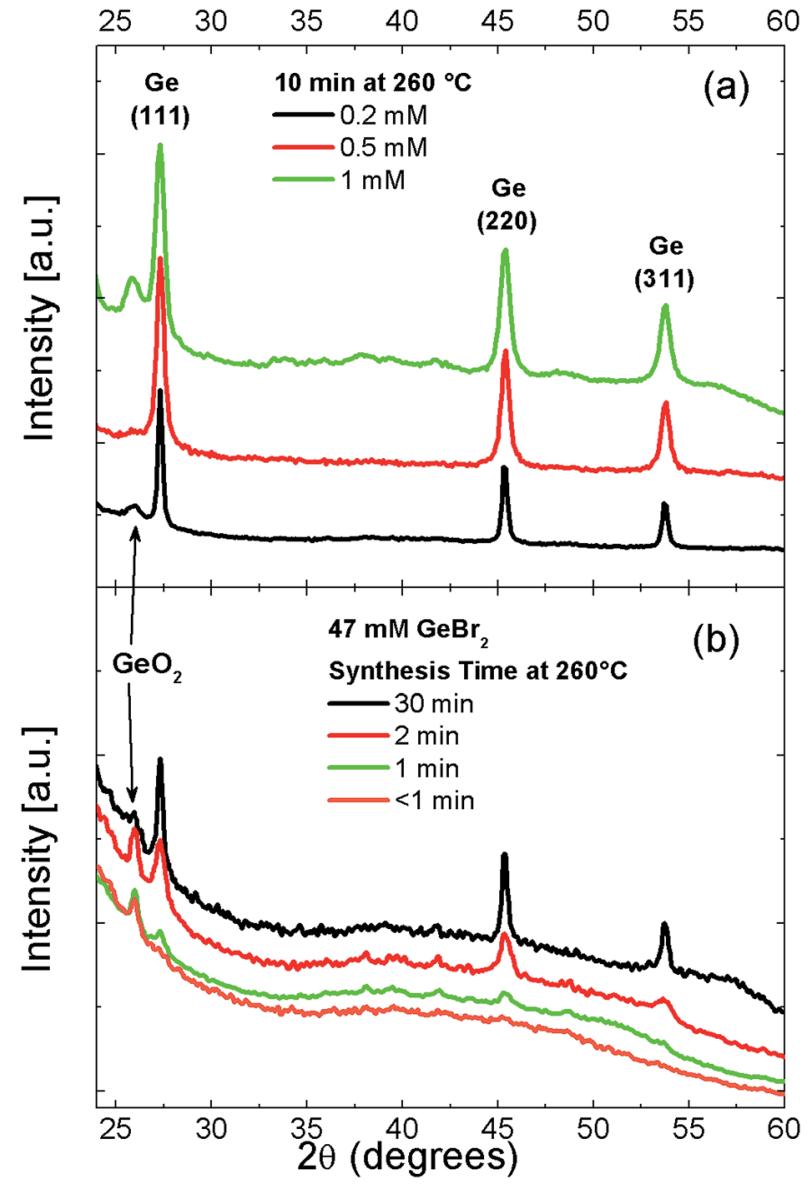

Fig. 2 XRD pattern of Ge NCs as a function of Ge precursor content (a) and synthesis time (b).

be detected for a threshold synthesis time shorter than one minute. In this case, only a broad background coming from the glassy substrate is present.

Besides the peaks relative to crystalline Ge, there are other peaks appearing in some of the XRD patterns, in particular for high amount of Ge precursor. Such peaks are uniquely related to rutile $\mathrm{GeO}_{2}$ (see Fig. $2 \mathrm{~S}$ in ESI $\dagger$ ). ${ }^{38}$ The presence of $\mathrm{GeO}_{2}$ is probably related to partial oxygen contamination of the precursor before the synthesis process, since $\mathrm{GeBr}_{2}$ is highly air sensitive. ${ }^{31}$ Such oxygen contamination can lead to the formation of a $\mathrm{GeO}_{2}$ shell around NCs or to $\mathrm{GeO}_{2}$ composites dispersed in the NC solution. Mild annealing pre-treatment of the $\mathrm{GeBr}_{2}$ powder allowed to restore the precursor to its original condition and removed $\mathrm{GeO}_{2}$-related contamination without any change of the FWHM of crystalline Ge-peak (see Fig. $3 \mathrm{~S}$ in ESI $\dagger$ ). This behavior gives evidence that the reaction pathway is not dramatically influenced by $\mathrm{GeO}_{2}$ contaminations in the precursor.

Fig. 3(a) shows the temporal growth evolution of Ge NCs synthesized at $260^{\circ} \mathrm{C}$ for the case of $47 \mathrm{mM}$ of Ge precursor, as extracted from XRD data. The NC mean size increases from 10 $\mathrm{nm}$ to about $25 \mathrm{~nm}$ in a characteristic time scale, $t_{\text {sat }}$, of about $300 \mathrm{~s}$. It is worth noting that the size extracted from XRD is compatible with the size distribution found by high resolution 

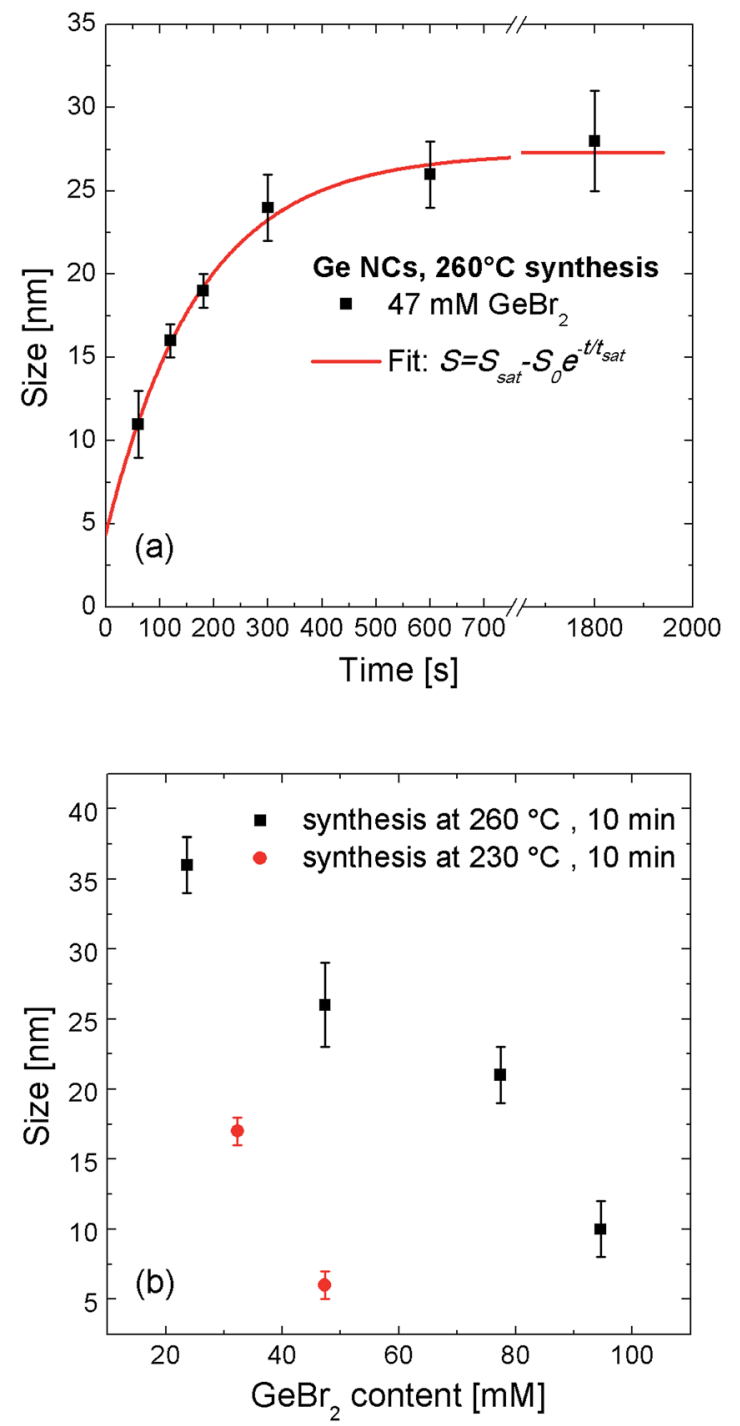

Fig. 3 Evolution of Ge NCs size as a function of synthesis time (a) and precursor content (b).

SEM analysis. The slightly larger value given by the latter could be attributed to a thin $\mathrm{GeO}_{2}$ shell covering the Ge $\mathrm{NC}$, as evidenced by the rutile $\mathrm{GeO}_{2}$ diffraction peak shown in Fig. 2. For synthesis times longer than 5 min NC growth clearly saturates to a value of almost $30 \mathrm{~nm}$. The experimental values of temporal NC growth are well fitted by the exponential relationship: $S=$ $S_{\text {sat }}-S_{0} \mathrm{e}^{-t / t_{\text {sat }}}$, where $S_{\text {sat }}$ is the NC size in the saturation regime and $S_{0}$ is the difference between $S_{\text {sat }}$ and $S_{\mathrm{cr}}^{\mathrm{exp}}$, which represents the critical size for NC growth. From the fitting we can extract a critical mean value for NC nucleation of $S_{\mathrm{cr}}^{\exp }=4.4 \pm 2.0 \mathrm{~nm}$.

Besides the role of synthesis time on NC growth, another main parameter is determined by the initial concentration of precursor, $C_{\mathrm{Ge}}$. As clearly shown in Fig. 3(b) the larger the Ge content, the smaller mean NCs size is. In particular, given a synthesis time of $10 \mathrm{~min}$ at $260{ }^{\circ} \mathrm{C}$, NC size decreases from a value of $35 \mathrm{~nm}$ for $24 \mathrm{mM}$ to $10 \mathrm{~nm}$ for $95 \mathrm{mM}$ of $\mathrm{GeBr}_{2}$. A similar trend is observed also for a synthesis temperature of 230 ${ }^{\circ} \mathrm{C}$, where NC size decreases from a value of about $17 \mathrm{~nm}$ for 32
$\mathrm{mM}$ to $6 \mathrm{~nm}$ for $47 \mathrm{mM}$ of $\mathrm{GeBr}_{2}$. We independently confirmed the trend of particle size vs. precursor concentration by Dynamic Light Scattering (DLS) technique. As shown in Fig. 4S of ESI, $\uparrow$ the DLS decay time from scattered photons by Ge particles decreases with the increase of the amount of dissolved Ge, indicating the presence of smaller particles in more concentrated solutions. No significant light scattering signal was observed for samples with drying pre-treated $\mathrm{GeBr}_{2}$ precursor, ruling out the presence of large $\mathrm{GeO}_{2}$ particles dispersed in solution. These findings reveal that synthesis time, Ge content and temperature, all are key-role parameters in the growth kinetic of colloidal NC.

\section{Interpretation and modelling}

In order to have a deeper understanding of the reported results, it is worth to separate the different phenomena taking place into the mechanisms of nucleation and growth of colloidal nanocrystals. For the simplified case of one nanoparticle dispersed in a solution of precursor atoms with concentration $C_{\mathrm{Ge}}$, the growth proceeds by two steps: (1) Ge atoms reach the nanoparticle surface from the bulk solution at a rate determined by the diffusion coefficient $D$; (2) Ge atoms react at the nanoparticle surface and are incorporated into it with a coefficient reaction rate $k_{\mathrm{r}}(T)=k_{0} \mathrm{e}^{-E_{\mathrm{r}} / k T}$, being $E_{\mathrm{r}}$ the activation energy of the reaction.

The size evolution of colloidal nanoparticles for different reaction and diffusion conditions were studied in detail in the works of Talapin et al. and Embden et al. ${ }^{32,33}$ They developed a model based on the size dependence of the activation energies for the growth and dissolution processes, which takes into account the reaction and diffusion rates. According to this model, the growth rate of a spherical particle with radius, $r$, immersed in a solution of atoms with concentration $C_{\mathrm{Ge}}$ can be written as: $:^{33}$

$$
\frac{\mathrm{d} r}{\mathrm{~d} t}=D V_{\mathrm{m}} C_{\text {flat }}^{0}\left[\frac{\frac{C_{\mathrm{Ge}}(x, y, t)}{C_{\mathrm{flat}}^{0}}-\exp \left(\frac{2 \gamma V_{\mathrm{m}}}{r R T}\right)}{r+D / k_{\mathrm{r}}}\right]
$$

where $V_{\mathrm{m}}$ is the molar volume of Ge, $C_{\text {flat }}^{0}$ is the solubility of Ge atoms in equilibrium with a flat interface and $\gamma$ is the specific surface energy of the growing nanoparticle (see Table 1). For the critical nucleation size, $S_{\mathrm{cr}}$, the rate of desorption is equal to the growth rate $\left(\mathrm{d} r / \mathrm{d} t=0\right.$ in eqn (1)) and $S_{\text {cr }}$ can be written as: ${ }^{32}$

$$
S_{\mathrm{cr}}^{\text {theor. }}=\frac{4 \gamma V_{\mathrm{m}}}{R T \ln \frac{C_{\mathrm{Ge}}}{C_{\mathrm{flat}}^{0}}}
$$

As $C_{\mathrm{Ge}}$ increases the critical size for NC nucleation reduces. This means that an increased concentration of Ge precursor dissolved in solution leads to a larger amount of smaller NCs. Such a prediction is confirmed by our experimental trend of NC size reported in Fig. 3(b). By substituting the experimental values of $C_{\mathrm{Ge}}$ used in the synthesis of our Ge $\mathrm{NC}$, we can determine values of $S_{\mathrm{cr}}^{\text {theor. }}$ ranging from $1.6 \mathrm{~nm}$ to $3.2 \mathrm{~nm}$ for 95 
Table 1 Values of boundary parameters used in the simulation of Ge NC growth

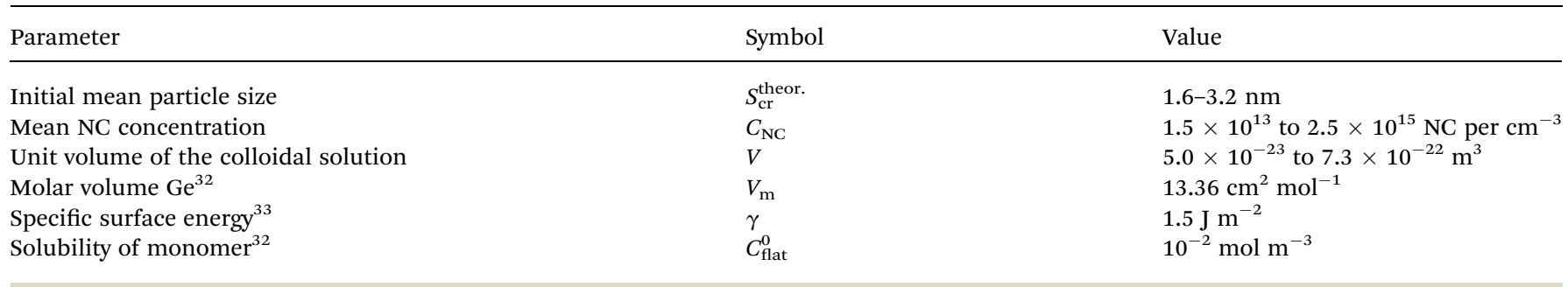

$\mathrm{mM}$ or $24 \mathrm{mM}$ of $\mathrm{GeBr}_{2}$, respectively. Indeed, such values of $S_{\mathrm{cr}}^{\text {theor. }}$ find a reasonable agreement, within the experimental error, with the value of $S_{\mathrm{cr}}^{\exp }$ extracted from the fitting of NC temporal growth evolution reported in Fig. 3(a).

In order to discern the type of growth regime, finite-elementanalysis calculations on NC growth evolution were computed by COMSOL software. We considered the simplified case of the growth of a single NC dispersed in a solution with a concentration of Ge atoms $C_{\mathrm{Ge}}$ and unit volume $V$. We imposed that at the initial stage $(t=0 \mathrm{~s})$ the NC has the critical size determined by eqn (2). Then, we made two fundamental assumptions only: (1) the whole amount of Ge precursor is involved in the formation of Ge NCs, i.e., at the end of the process no dissolved Ge will remain in solution; (2) no significant Ostwald ripening or coalescence effects take place during the growth process. Both these assumptions find conceptual agreement with theoretical studies on NC growth evolution in colloidal solution, ${ }^{32,33}$ allowing to describe this process through two parameters only: amount of Ge precursor and synthesis temperature. Indeed, both the above assumptions are compatible also with our experimental results. The first one is justified by the timescale used in the synthesis process. This was chosen long enough to allow the complete dissolution and incorporation of all Ge atoms toward the growing NCs, as also confirmed by the saturation of NC size reported in Fig. 3(a). Our latter assumption can be justified by the relatively sparse distribution of Ge NCs existing in our experimental conditions. In fact, the average NC concentration in the whole colloidal solution can be estimated through the formula $C_{\mathrm{NC}}=C_{\mathrm{Ge}}\left(\frac{4}{3} \pi r^{3} \rho_{\mathrm{Ge}}\right)^{-1}$ (where $\rho_{\mathrm{Ge}}$ is the Ge atomic density). In this way, it is possible to estimate a mean NC concentration going from $1.5 \times 10^{13} \mathrm{NC}$ per $\mathrm{cm}^{-3}$ for $24 \mathrm{mM}$ to $2.5 \times 10^{15} \mathrm{NC}$ per $\mathrm{cm}^{-3}$ for $95 \mathrm{mM}$ of $\mathrm{GeBr}_{2}$. The corresponding mean distance between two NC, $d[d$ $=\left(C_{\mathrm{NC}}\right)^{-1 / 3}-2 r$, defining the unit volume $V$ of solution for each $\mathrm{NC}$ ], increases from around $60 \mathrm{~nm}$ to $370 \mathrm{~nm}$ as $C_{\mathrm{NC}}$ goes from $2.5 \times 10^{15} \mathrm{NC}$ per $\mathrm{cm}^{-3}$ to $1.5 \times 10^{13} \mathrm{NC}$ per $\mathrm{cm}^{-3}$, as listed in Table 1 .

The boundary conditions in Table 1 were used as starting values to describe NC size evolution in our system. Our model calculates the time-dependent evolution of $C_{\mathrm{Ge}}$ in each point defined by the applied spatial mesh. After each time step, the spatial concentration of monomer still present in the solution is analytically calculated in our model and is substituted into the time dependent equation of the radius, which is written as:

$$
\begin{aligned}
r\left(t_{i}\right) & =r\left(t_{i-1}\right)+\frac{\mathrm{d} r}{\mathrm{~d} t} \Delta t \\
& =r\left(t_{i-1}\right)+D V_{\mathrm{m}} C_{\mathrm{flat}}^{0} \frac{\left[\frac{C_{\mathrm{Ge}}\left(x, y, t_{i-1}\right)}{C_{\mathrm{flat}}^{0}}-\exp \left(\frac{2 \gamma V_{\mathrm{m}}}{r\left(t_{i-1}\right) R T}\right)\right]}{r\left(t_{i-1}\right)+D / k_{\mathrm{r}}} \Delta t
\end{aligned}
$$

We studied the growth process within a time range between 0 and 1800 seconds, with time-step, $\Delta t$, of $0.01 \mathrm{~s}$. The concentration of Ge in the solution was calculated after each time step and was substituted into eqn (1) and (3) to calculate the growth rate in the next step. In this regard, the only free parameter in our simulation is the a-dimensional quantity $K=\frac{R T}{2 \gamma V_{\mathrm{m}}} \frac{D}{k_{\mathrm{r}}}$, which gives information on the growth regime. If $K \ll 1$, the growth kinetic is limited by the low diffusivity of Ge atoms towards the nanoparticle surface. On the contrary, if $K \gg 1$, then the growth kinetic is limited by the reaction of atoms at the nanoparticle surface (low incorporation rate). The numerical parameters used in our simulations are presented in Table 2.

Fig. 4 shows the simulation of size evolution of a single nanoparticle as a function of the synthesis time, in the case of an initial precursor content of $47 \mathrm{mM}$ and a temperature of 260 ${ }^{\circ} \mathrm{C}$. Note that at the initial stage $(t=0 \mathrm{~s})$ the nanoparticle is represented as a (white) circular dot having a critical size of 2.6 $\mathrm{nm}$, according to eqn (2). At this stage, the largest part of Ge precursor is still dissolved in the solution, while only a small fraction is used to form the initial Ge nuclei. When the growth starts, NC size gets bigger because of diffusion and incorporation of Ge atoms. In this regard, NC can be imagined as a sink for the diffusing Ge atoms. As the NC growth proceeds, the concentration of Ge atoms in the solutions is lowered, as shown in Fig. 4. This leads to a saturation of the NC growth, since after a certain time no more atoms are available in the solution for sustaining a further NC growth. This is exactly what we experimentally observed in the case of $47 \mathrm{mM} \mathrm{GeBr}_{2}$ as reported in Fig. 3(a).

The simulation results of the NC growth versus time are reported in Fig. 5. By varying the ratio $D / k_{\mathrm{r}}$ is possible to satisfactorily fit the experimental values of the NC growth. As shown in Fig. 5(a) for the case of $C_{\mathrm{Ge}}=47 \mathrm{mM}$ at $260{ }^{\circ} \mathrm{C}$, a very good agreement between the $\mathrm{NC}$ growth simulation and the experimental results is found for a growth parameter of $K=10$. Noticeably, maintaining the same value of $K$ allows getting a reasonable agreement with experiments whatever is the initial 
Table 2 Range of the values of the growth parameters used in the simulation of Ge NC growth

\begin{tabular}{lll}
\hline Parameter & Symbol & Value \\
\hline Initial monomer over saturation & $C_{\mathrm{Ge}} / C_{\mathrm{flat}}^{0}$ & $2350-9500$ \\
Reaction coefficient & $k_{\mathrm{r}}$ & $8.0 \times 10^{-11}$ to $9.8 \times 10^{-10} \mathrm{~m} \mathrm{~s}^{-1}$ \\
Diffusion coefficient & $D$ & $8.0 \times 10^{-17}$ to $9.2 \times 10^{-17} \mathrm{~m}^{2} \mathrm{~s}^{-1}$ \\
Growth parameter & $K=\frac{R T}{2 \gamma V_{\mathrm{m}}} \frac{D}{k_{\mathrm{r}}}$ & $1-200$
\end{tabular}
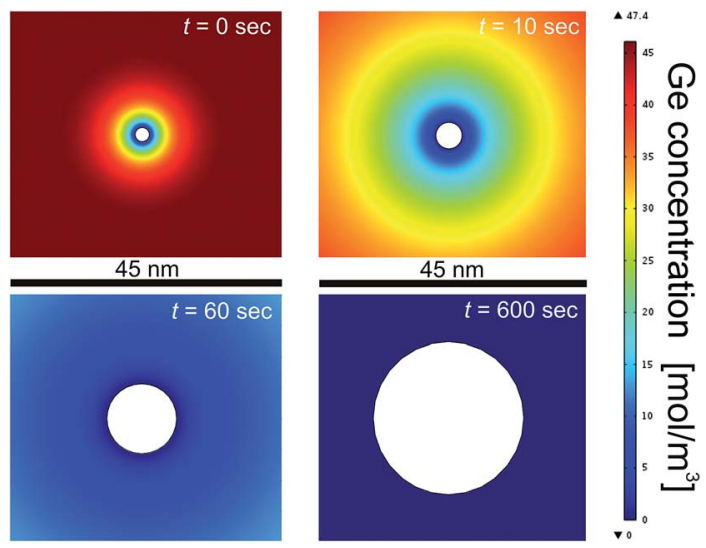

Fig. 4 Simulation of NC growth evolution for a colloidal solution of Ge atoms dissolved in oleylamine in the case of initial precursor content of $47 \mathrm{mM}$ and $260^{\circ} \mathrm{C}$ synthesis temperature.

Ge concentration used in the 24-95 $\mathrm{mM}$ range. This important result highlights that Ge NC growth kinetics, under the investigated conditions, are always in a regime controlled by the incorporation reaction at the NC surface. Moreover, both simulation and experimental values evidence that NC size decreases with the increase of the Ge concentration. Finally, it is possible to observe that the temporal evolution of NC growth saturation is highly dependent on the Ge content. While for a low amount of precursor the NC size starts to saturate for $t>$ $600 \mathrm{~s}$, a characteristic saturation time $<100 \mathrm{~s}$ is found for the higher concentrations. This behaviour is compatible with a reaction-limited regime. In fact, the higher density of nuclei induces a quicker consumption of dissolved $\mathrm{Ge}$ atoms and consequently a faster saturation time of the NC growth. $\mathrm{GeO}_{2}$ contamination arising from a partial contamination of $\mathrm{GeBr}_{2}$ precursor can still have some influences in the final pathway of Ge NCs growth. In the worst scenario of a large oxidized precursor, only a lower amount of Ge atoms would be involved in the growth of Ge NC, since oxidized Ge atoms will probably not contribute to Ge NC growth. Since we did not include oxidation effects in our model, and we assumed all the Ge atoms participating to $\mathrm{NC}$ growth, the results of our simulations would give an underestimated value of NC size. This is actually what happens in our simulations for the case of $77 \mathrm{mM}$ and 95 $\mathrm{mM}$ of Ge, as shown in Fig. 5(a). This behavior remarks the general validity of our model in describing the growth kinetics of Ge NCs. Besides this underestimation of NC size, the main trends and conclusions on the effects of Ge amount and growth parameter remain still valid.
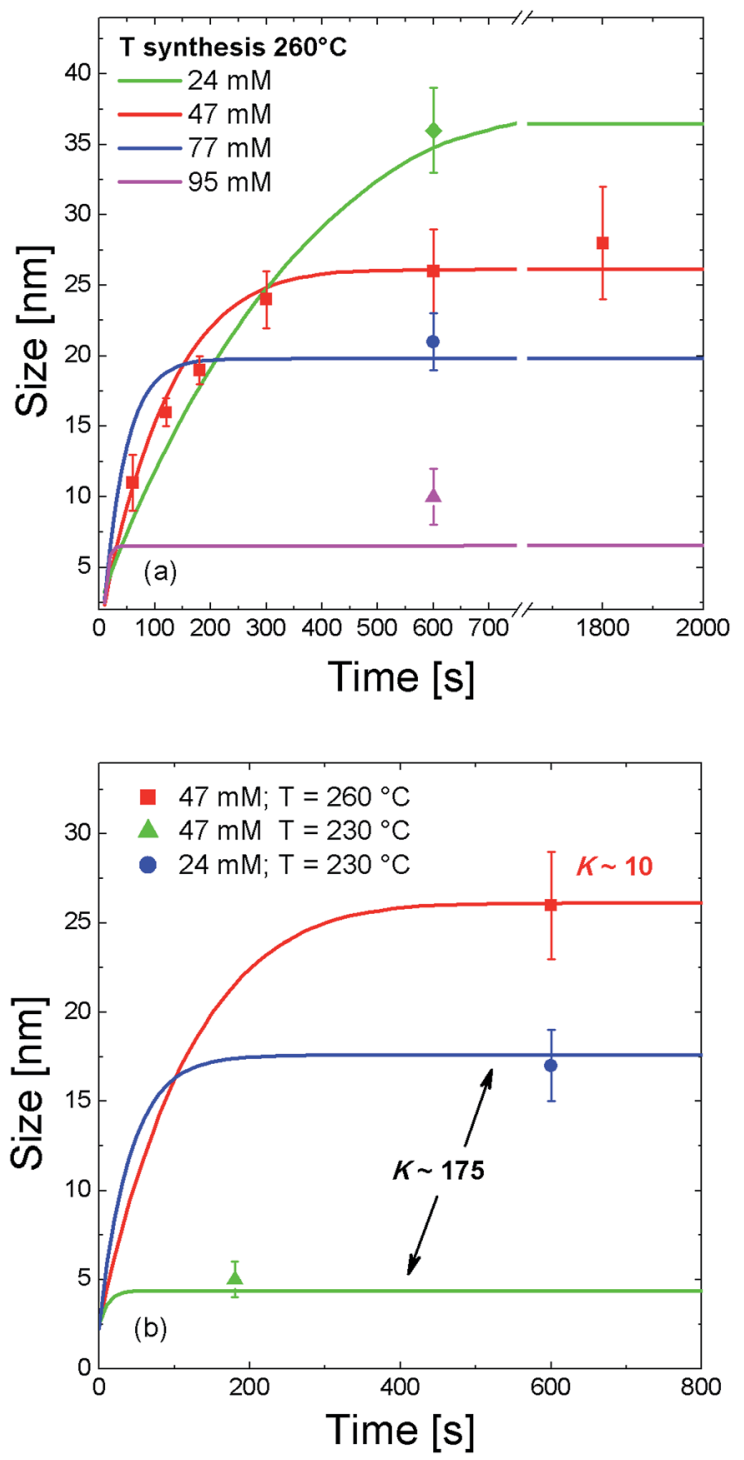

Fig. 5 Simulation of the temporal size evolution of colloidal Ge NC growth. The effects of Ge content (a) and synthesis temperature (b) are evidenced.

Moreover, both diffusion and reaction processes can be highly dependent on the temperature. This means that a change of the synthesis temperature can lead to a different growth regime for NC. Fig. 5(b) elucidates this behaviour. As already shown in Fig. 3(b), a decrease of the temperature from $260^{\circ} \mathrm{C}$ to $230{ }^{\circ} \mathrm{C}$ results in the formation of smaller NCs. In this case 
a good agreement between experimental results and NC growth simulation is found for a growth parameter condition $K=175$. Again, it is worth to note that for the same value of $K$, the simulation model nicely describes the experimental results for two different values of Ge content at $230{ }^{\circ} \mathrm{C}$. More importantly, this parameter increases of almost a factor 20 when the synthesis temperature decreases from $260{ }^{\circ} \mathrm{C}$ to $230{ }^{\circ} \mathrm{C}$. This means that for a lower synthesis temperature the NC growth becomes strongly limited by the reaction process. In other words, the process of Ge incorporation into the NC is highly dependent on the synthesis temperature and by decreasing the temperature it decreases at a rate much faster than the atom diffusion process. By considering the values of $k_{\mathrm{r}}$ extracted from the simulation at $230{ }^{\circ} \mathrm{C}\left(k_{\mathrm{r}}=8.7 \times 10^{-11} \mathrm{~m} \mathrm{~s}^{-1}\right)$ and at $260{ }^{\circ} \mathrm{C}$ $\left(k_{\mathrm{r}}=9.8 \times 10^{-10} \mathrm{~m} \mathrm{~s}^{-1}\right)$, together with its temperature dependence $k_{\mathrm{r}}(T)=k_{0} \mathrm{e}^{-E_{\mathrm{r}} / k T}$, we determined an activation energy of the reaction $E_{\mathrm{r}} \sim 0.7 \mathrm{eV}$. Such a quantity, which represents the energy barrier to the incorporation of Ge atoms into the NC surface, is comparable with the typical bonding energy of atoms onto the surface of a Ge NC. ${ }^{39}$ Therefore, synthesis temperature intimately controls the NC growth process regime through the reaction processes at the NC surface. These results add further understanding of the key-role parameters in the synthesis of colloidal Ge NCs.

\section{Light harvesting performances}

After the fundamental studies performed on the synthesis of colloidal Ge NCs, we tested their light harvesting performances. Transmission $\left(I_{\mathrm{T}}\right)$ spectra in the 400-1100 nm range for Ge NC dispersed $(1: 3)$ in toluene are reported in Fig. 6(a). The presence of Ge NCs induces a clear decrease of $I_{\mathrm{T}}$ in the visible range, which is attributable to direct light absorption from Ge NC.,40 This decrease is not related to absorption from toluene and oleylamine reference solution, being optically transparent in the investigated spectral range (see Fig. 5S in ESI $\dagger$ ).

Finally, we tested the potential capability of photo-carrier collection in prototypal devices with colloidal Ge NCs. Till now, there have been some attempts of colloidal Ge-NC for photovoltaic or transistor devices by spinning solutions of colloidal Ge NC to grow a porous Ge-NC film. ${ }^{\mathbf{1 4 , 4 1 , 4 2}}$ This approach is typically limited by a poor conductivity of the absorbing media and also suffers from the loss of quantum effects in NCs assembly film. In order to improve the charge carrier transport performance, we mixed Ge NCs in PEDOT:PSS polymer to form a hybrid conductive film. Such a film was spin on interdigitated $\mathrm{Au}$ electrodes, as shown in the schematic of Fig. 6(b). More remarkably, Fig. 6(b) shows the current of the device during repetitive ON/OFF switching of light illumination. A clear increase and decrease of the photo-current is observed for the device with Ge NCs as a response of the ON/OFF illumination, respectively. Control device without Ge NCs immersed in the PEDOT:PSS film does not show any response to the light excitation. A photoelectric device, based on the conjunction of colloidal Ge NCs with a conjugated poly(3hexylthiophene) polymer (P3HT), was recently developed by Xue and co-workers. ${ }^{13}$ However, P3HT is an expensive polymer,
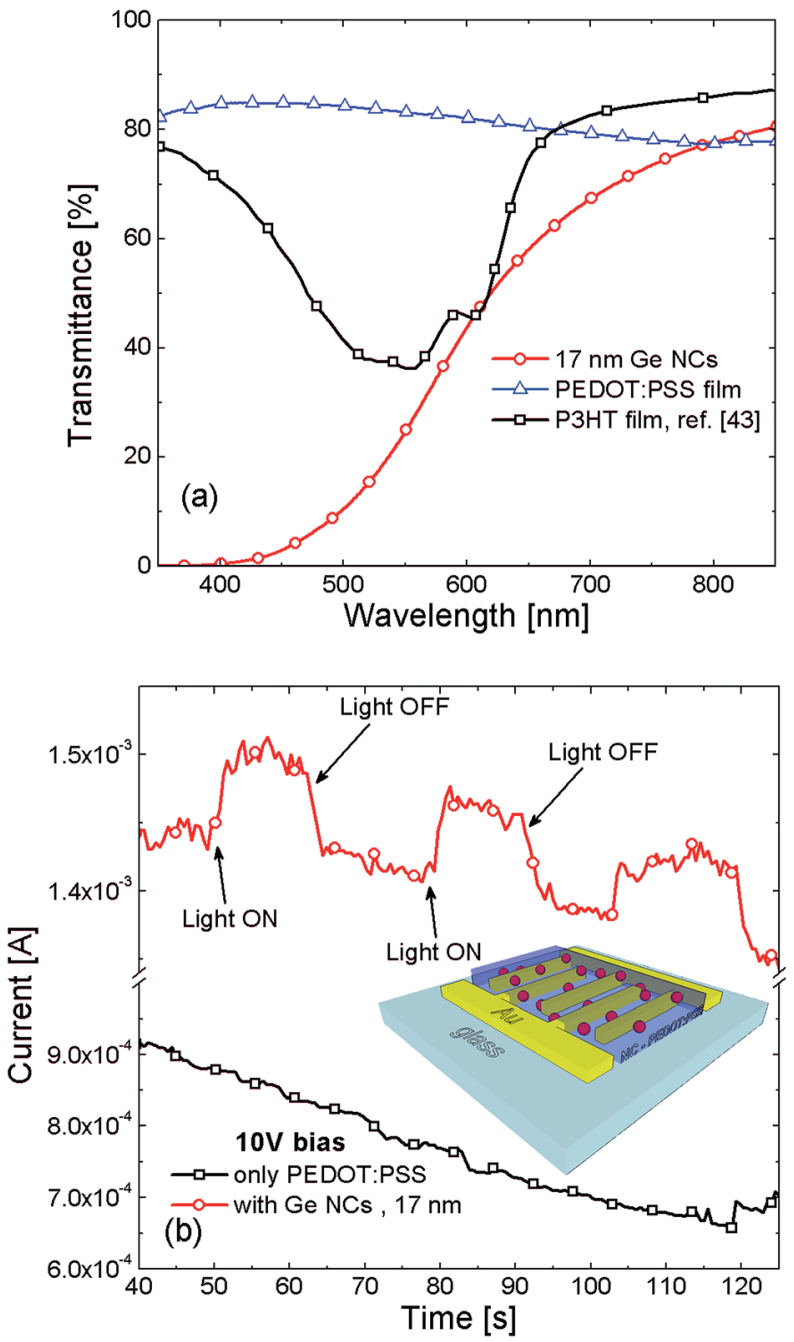

Fig. 6 Transmittance spectra of colloidal Ge NC solution (dispersed $1: 3$ in toluene) (a). Light ON/OFF switching characteristics of a hybrid device with Ge NC immersed in PEDOT:PSS film (b). A schematic of the device is reported in the figure.

which also strongly absorbs light in the VIS region, as reported in Fig. 6(a) for a $78 \mathrm{~nm}$ P3HT thin film. ${ }^{43}$ This effect hides the contribution of Ge NCs in the light harvesting process. In our case, PEDOT:PSS is intentionally used for its lower cost and transparency in the whole VIS-NIR range. As shown in Fig. 6(a), the bare PEDOT:PSS film ( $\sim 200 \mathrm{~nm}$ thickness) does not contribute to any photo-charge generation upon illumination, being its absorption negligible with respect to Ge NCs. These results definitively highlight the key-role of Ge NCs as light absorbing centers. The carriers photo-generated into the Ge NCs are then transported within the conductive polymer layer reaching the device contacts. While it was previously demonstrated that Ge NCs (produced by UHV techniques) can be profitably used as efficient photo-sensitizer in light detection devices, ${ }^{\mathbf{4}, 5}$ here we show that solution synthesized Ge NCs have similar potentiality for light detection. Indeed, through a proper control of size and growth parameters, it would be possible to effectively exploit QCE and develop tunable, efficient and lowcost photodetectors compatible with Si technology. 


\section{Conclusions}

In conclusion, we reported a wide investigation on the role of growth parameters in the synthesis process of colloidal Ge NCs. Colloidal Ge NCs were synthesized through one-pot heat up method from the reduction of $\mathrm{GeBr}_{2}$ precursor dissolved in oleylamine at $230-260{ }^{\circ} \mathrm{C}$. Through variation of synthesis time, temperature and precursor concentration, it is possible to effectively tune the NC size in the 5-35 $\mathrm{nm}$ range. We experimentally verified that NC size increases with the synthesis time, saturating after a characteristic time of about $300 \mathrm{~s}$. Moreover, NC growth strongly depends on the precursor content, resulting in the production of a larger amount of smaller NC when the initial precursor content is increased. Basing on our experimental data, we simulated the growth process of Ge NCs in solution as a function of the precursor content and synthesis temperature. We demonstrated that in the investigated conditions, the kinetics of NC growth is controlled by a reactionlimited regime. In particular, the temperature of synthesis process strongly controls the NC growth regime, resulting in a strongly-limited reaction regime for a synthesis temperature of $230{ }^{\circ} \mathrm{C}$. Finally, we proved the light detection capability of our colloidal Ge NCs. Transmittance spectroscopy demonstrated the light absorption features in the VIS region of Ge NCs. Then, we exploited the light absorption from colloidal Ge NCs in a hybrid photoelectric device with Ge NCs embedded in PEDOT:PSS conductive polymer. The clear current increase and decrease during ON/OFF switching of light illumination proves the chance to use colloidal Ge NCs for light detection purposes. These results put new understanding for a more reliable control of colloidal NC growth and the development of low-cost devices.

\section{Acknowledgements}

The authors thank G. Panté (MATIS CNR-IMM) for expert technical assistance and prof. R. Reitano for stimulating discussion. This work was funded by project ENERGETIC PON02_00355_3391233/F1.

\section{References}

1 F. Priolo, T. Gregorkiewicz, M. Galli and T. F. Krauss, Silicon nanostructures for photonics and photovoltaics, Nat. Nanotechnol., 2014, 9, 19.

2 G. Konstantatos and E. H. Sargent, Nanostructured materials for photon detection, Nat. Nanotechnol., 2010, 5, 391.

3 S. Cosentino, A. M. Mio, E. G. Barbagiovanni, R. Raciti, R. Bahariqushchi, M. Miritello, G. Nicotra, A. Aydinli, C. Spinella, A. Terrasi and S. Mirabella, The role of the interface in germanium quantum dots: when not only size matters for quantum confinement effects, Nanoscale, 2015, 7, 11401.

4 S. Cosentino, E. G. Barbagiovanni, I. Crupi, M. Miritello, G. Nicotra, C. Spinella, D. Pacifici, S. Mirabella and A. Terrasi, Size dependent light absorption modulation and enhanced carrier transport in germanium quantum dots devices, Sol. Energy Mater. Sol. Cells, 2015, 135, 22-28.
5 C. Y. Chien, W. T. Lai, Y. J. Chang, C. C. Wang, M. H. Kuo and P. W. Li, Size tunable Ge quantum dots for near-ultraviolet to near-infrared photosensing with high figures of merit, Nanoscale, 2014, 6, 5303.

6 S. Cosentino, S. Mirabella, M. Miritello, G. Nicotra, R. L. Savio, F. Simone, C. Spinella and A. Terrasi, The role of the surfaces in the photon absorption in Ge nanoclusters embedded in silica, Nanoscale Res. Lett., 2011, $6,135$.

7 S. Lee, S. Huang, G. Conibeer and M. Green, Lateral growth of Ge nanocrystals in a thin Ge-rich silicon nitride layer, $J$. Cryst. Growth, 2013, 383, 36.

8 S. Cosentino, E. Sungur Ozen, R. Raciti, A. M. Mio, G. Nicotra, F. Simone, R. Turan, A. Terrasi, A. Aydinli and S. Mirabella, Light harvesting with Ge quantum dots embedded in $\mathrm{SiO}_{2}$ or $\mathrm{Si}_{3} \mathrm{~N}_{4}$, J. Appl. Phys., 2014, 115, 043103.

9 D. J. Lockwood, N. L. Rowell, I. Berbezier, G. Amiard, A. Ronda and M. Faustini, Photoluminescence Efficiency of Self-Assembled Ge Nanocrystals, J. Electrochem. Soc., 2009, 156, H913.

10 X. Wang, Z. M. Jiang, H. J. Zhu, F. Lu, D. Huang, C. W. Hu, Y. Chen, Z. Zhu, T. Yao and X. Liu, Germanium dots with highly uniform size distribution grown on $\mathrm{Si}(100)$ substrate by molecular beam epitaxy, Appl. Phys. Lett., 1997, 71, 3543.

11 S. Mirabella, S. Cosentino, A. Gentile, G. Nicotra, N. Piluso, L. V. Mercaldo, F. Simone, C. Spinella and A. Terrasi, Matrix role in Ge nanoclusters embedded in $\mathrm{Si}_{3} \mathrm{~N}_{4}$ or $\mathrm{SiO}_{2}$, Appl. Phys. Lett., 2012, 101, 011911.

12 B. Sun, G. Zou, X. Shen and X. Zhang, Exciton dissociation and photovoltaic effect in germanium nanocrystals and poly(3-hexylthiophene) composites, Appl. Phys. Lett., 2009, 94, 233504.

13 D. J. Xue, J. J. Wang, Y. Q. Wang, S. Xin, Y. G. Guo and L. J. Wan, Facile synthesis of germanium nanocrystals and their application in organic-inorganic hybrid photodetectors, Adv. Mater., 2011, 23, 3704.

14 C. P. Church, E. Muthuswamy, G. Zhai, S. M. Kauzlarich and S. A. Carter, Quantum dot $\mathrm{Ge} / \mathrm{TiO}_{2}$ heterojunction photoconductor fabrication and performance, Appl. Phys. Lett., 2013, 94, 233504.

15 A. Karatutlu, M. Song, A. P. Wheeler, O. Ersoy, W. R. Little, Y. Zhang, P. Puech, F. S. Boi, Z. Luklinskae and A. V. Sapelkina, Synthesis and structure of free-standing germanium quantum dots and their application in live cell imaging, $R S C A d v$., 2015, 5, 20566.

16 D. D. Vaughn II and R. E. Schaak, Synthesis properties and applications of colloidal germanium and germaniumbased nanomaterials, Chem. Soc. Rev., 2013, 42, 2861.

17 B. R. Taylor, S. M. Kauzlarich, H. W. H. Lee and G. R. Delgado, Solution synthesis of germanium nanocrystals demonstrating quantum confinement, Chem. Mater., 1998, 10, 22.

18 B. R. Taylor, S. M. Kauzlarich, G. R. Delgado and H. W. H. Lee, Solution synthesis and characterization of quantum confined Ge nanoparticles, Chem. Mater., 1999, 11, 2493. 
19 R. S. Tanke, S. M. Kauzlarich, T. E. Patten, K. A. Pettigrew, D. L. Murphy, M. E. Thompson and H. W. H. Lee, Synthesis of germanium nanoclusters with reversibly attached functional groups: Acetals, alcohols, esters, and polymers, Chem. Mater., 2003, 15, 1682.

20 J. P. Wilcoxon, P. P. Provencio and G. A. Samara, Synthesis and optical properties of colloidal germanium nanocrystals, Phys. Rev. B: Condens. Matter Mater. Phys., 2001, 64, 035417.

21 P. Wilcoxon, P. P. Provencio and G. A. Samara, Erratum: synthesis and optical properties of colloidal germanium nanocrystals, Phys. Rev. B: Condens. Matter Mater. Phys., 2007, 76, 199904(E).

22 J. H. Warner and R. D. Tilley, Synthesis of water-soluble photoluminescent germanium nanocrystals, Nanotechnology, 2006, 17, 3745.

23 X. M. Lu, B. A. Korgel and K. P. Johnston, High Yield of Germanium Nanocrystals Synthesized from Germanium Diiodide in Solution, Chem. Mater., 2005, 17, 6479.

24 D. Carolan and H. Doyle, Size and emission color tuning in the solution phase synthesis of highly luminescent germanium nanocrystals, J. Mater. Chem. C, 2014, 2, 3562.

25 D. Gerion, N. Zaitseva, C. Saw, M. F. Casula, S. Fakra, T. Van Buuren and G. Galli, Solution Synthesis of Germanium Nanocrystals: Success and Open Challenges, Nano Lett., 2004, 4, 597.

26 H. P. Wu, M. Y. Ge, C. W. Yao, Y. W. Wang, Y. W. Zeng, L. N. Wang, G. Q. Zhang and J. Z. Jiang, Blue emission of Ge nanocrystals prepared by thermal decomposition, Nanotechnology, 2006, 17, 5339.

27 D. D. Vaughn II, J. F. Bondi and R. E. Schaak, Colloidal Synthesis of Air-Stable Crystalline Germanium Nanoparticles with Tunable Sizes and Shapes, Chem. Mater., 2010, 22, 6103.

28 E. Muthuswamy, A. S. Iskandar, M. M. Amador and S. M. Kauzlarich, Facile Synthesis of Germanium Nanoparticles with Size Control: Microwave versus Conventional Heating, Chem. Mater., 2013, 25, 1416.

29 S. C. Codoluto, W. J. Baumgardner and T. Hanrath, Fundamental aspects of nucleation and growth in the solution-phase synthesis of germanium nanocrystals, CrystEngComm, 2010, 12, 2903.

30 T. K. Purkait, A. K. Swarnakar, G. B. De Los Reyes, F. A. Hegmann, E. Rivard and J. G. C. Veinot, One-pot synthesis of functionalized germanium nanocrystals from a single source precursor, Nanoscale, 2015, 7, 2241.
31 D. C. Lee, J. M. Pietryga, I. Robel, D. J. Werder, R. D. Schaller and V. I. Klimov, Colloidal Synthesis of Infrared-Emitting Germanium Nanocrystals, J. Am. Chem. Soc., 2009, 131, 3436.

32 D. V. Talapin, A. L. Rogach, M. Haase and H. Weller, Evolution of an Ensemble of Nanoparticles in a Colloidal Solution: Theoretical Study, J. Phys. Chem. B, 2001, 105, 12278.

33 J. van Embden, J. E. Sader, M. Davidson and P. Mulvaney, Evolution of Colloidal Nanocrystals: Theory and Modeling of their Nucleation and Growth, J. Phys. Chem. C, 2009, 113, 16342.

34 P. Scherrer, Bestimmung der Größe und der inneren Struktur von Kolloidteilchen mittels Röntgenstrahlen, Nachr. Ges. Wiss. Göttingen, 1918, 26, 98.

35 Y. Xia and J. Ouyang, PEDOT:PSS films with significantly enhanced conductivities induced by preferential solvation with cosolvents and their application in polymer photovoltaic cells, J. Mater. Chem., 2011, 21, 4927.

36 S. Mourdikoudis and L. M. Liz-Marzań, Oleylamine in Nanoparticle Synthesis, Chem. Mater., 2013, 25, 1465.

37 D. A. Ruddy, J. C. Johnson, E. R. Smith and N. R. Neale, Size and Bandgap Control in the Solution-Phase Synthesis of Near-Infrared-Emitting Germanium Nanocrystals, ACS Nano, 2010, 12, 7459.

38 S. L. Sewell, R. D. Rutledge and D. W. Wright, Versatile biomimetic dendrimer templates used in the formation of $\mathrm{TiO}_{2}$ and $\mathrm{GeO}_{2}$, Dalton Trans., 2008, 29, 3857.

39 D. Tomanek and M. A. Schluter, Structure and bonding of small semiconductor clusters, Phys. Rev. B: Condens. Matter Mater. Phys., 1987, 36, 1208.

40 A. Lepadatu, I. Stavarache, M. L. Ciurea and V. Iancu, The influence of shape and potential barrier on confinement energy levels in quantum dots, J. Appl. Phys., 2010, 107, 033721.

41 Z. C. Holman, C.-Y. Liu and U. R. Kortshagen, Germanium and Silicon Nanocrystal Thin-Film Field-Effect Transistors from Solution, Nano Lett., 2010, 10, 2661.

42 L. M. Wheeler, A. W. Nichols, B. D. Chernomordik, N. C Anderson, M. C. Beard and N. R. Neale, All-Inorganic Germanium Nanocrystal Films by Cationic Ligand Exchange, Nano lett., 2016, 16(3), 1949-1954.

43 V. N. Peters, T. U. Tumkur, G. Zhu and M. A. Noginov, Control of a chemical reaction (photodegradation of the p3ht polymer) with nonlocal dielectric environments, Sci. Rep., 2015, 5, 14620. 